\title{
Increased Apoptosis, Altered Oxygen Signaling, and Antioxidant Defenses in First-Trimester Pregnancies with High-Resistance Uterine Artery Blood Flow
}

Karin Leslie, ${ }^{* \dagger}$ Guy StJ. Whitley, ${ }^{*}$ Florian Herse ${ }^{\ddagger}$ Ralf Dechend, ${ }^{\dagger}$ Sandra V. Ashton, ${ }^{*}$ Ken Laing, ${ }^{\S}$ Baskaran Thilaganathan, ${ }^{\dagger}$ and Judith E. Cartwright*

From the Institutes of Cardiovascular and Cell Sciences* and Infection and Immunity, ${ }^{\S}$ St. George's University of London, London, United Kingdom; the Fetal Medicine Unit, ${ }^{\dagger}$ St. George's Hospital, London, United Kingdom; and the Experimental and Clinical Research Center, ${ }^{\ddagger}$ a Joint Cooperation between the Max-Delbrück Center for Molecular Medicine and the Charité Medical Faculty, Berlin, Germany

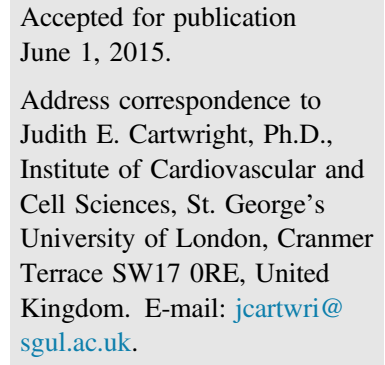

\begin{abstract}
The mechanisms of deficient placentation in the first trimester remain poorly understood, although apoptosis, hypoxia, and oxidative stress have been implicated. High uterine artery Doppler resistance indexes (RIs) are predictive of placental complications of pregnancy, such as preeclampsia, fetal growth restriction, and stillbirth. We provide evidence that even in the first trimester, pregnancies with high uterine artery Doppler RI demonstrate alterations in placental gene and protein expression. Apoptosis was significantly higher in high RI placental tissue, as determined by Western blot analysis of cleaved poly (ADP-ribose) polymerase and caspase 3. Protein expression of the trophoblast survival factor insulin-like growth factor-2 was significantly lower. Both high and normal RI placentas showed evidence of hypoxia and oxidative stress with expression of hypoxia-inducible factors $1 \alpha$ and $2 \alpha$, heat shock protein 70, presence of nitrotyrosine residues, and lipid peroxidation. We observed no exaggerated placental hypoxia or oxidative stress associated with high RI pregnancies. High RI placental tissue demonstrated an altered balance of antioxidant enzyme activity. Hypoxia and oxidative stress appear to be a physiological state in early pregnancy; our data did not support the hypothesis that they are associated with deficient placentation in the first trimester. Higher levels of apoptosis, reduced insulin-like growth factor-2 expression, and altered antioxidant defenses may contribute to abnormal placentation and the later development of pregnancy complications, such as preeclampsia, fetal growth restriction, and stillbirth. (Am J Pathol 2015, 185: 2731-2741; http://dx.doi.org/ 10.1016/j.ajpath.2015.06.020)
\end{abstract}

Successful outcome of pregnancy is dependent on placental sufficiency, namely successful implantation and remodeling of the maternal uterine spiral arteries in early pregnancy. Disorders of pregnancy related to deficient placentation include preeclampsia (PE), fetal growth restriction (FGR), abruption, and stillbirth. ${ }^{1-3}$ The pathophysiology of these placental obstetric disorders remains unclear, although advances in our understanding have been made in recent years. ${ }^{4,5} \mathrm{PE}$, in particular, is a major cause of maternal morbidity and mortality worldwide and occurs in $2 \%$ to $5 \%$ of pregnancies. ${ }^{6}$
Evidence of oxidative stress, hypoxia, and altered antioxidant defenses have been demonstrated in placental studies of pregnancies affected by PE. ${ }^{7,8}$ Apoptosis, which may result from increased cell stress, has also been implicated in the pathogenesis of deficient placentation, with higher levels of placental apoptosis in PE and FGR. ${ }^{9,10}$ However, study of the placenta after delivery has limited value because by that

Supported by the National Institute for Health Research Clinical Lectureship (K.L.) and by Action Medical Research UK grant SP4577.

Disclosures: None declared. 
time the disease process has progressed to the point that delivery is indicated. These studies, although highly suggestive that pathways involving placental hypoxia, oxidative stress, and apoptosis are involved in diseases of placental origin, are unable to answer the question of whether they occur as a response to the disease or are truly causal in the pathogenesis. If we are to institute treatments to ameliorate, or ideally prevent, the consequences of poor placentation, then an understanding of the pathophysiology in the first trimester is necessary.

Uterine artery Doppler (UtAD) ultrasound has been shown to be predictive of placental complications in pregnancy. ${ }^{11,12}$ It has a greater ability to predict preterm PE and PE associated with FGR than term disease, which may not have such a clear placental origin. ${ }^{13-15}$ High-resistance UtAD indices in the first trimester are associated with decreased endovascular trophoblast invasion ${ }^{16}$ and an increased risk of placental complications of pregnancy. ${ }^{11}$ We have previously shown functional differences in highresistance pregnancies in trophoblast sensitivity to apoptotic stimuli and decidual natural killer cell function that would potentially have a detrimental impact on placentation. ${ }^{17,18}$ We hypothesized that placentas from pregnancies with high-resistance UtAD flow would demonstrate alterations in apoptosis, oxidative stress, antioxidant defenses, and placental oxygen signaling.

\section{Materials and Methods}

\section{First-Trimester UtAD and Pregnancy Outcome in Continuing Pregnancies}

This was a prospective, observational study of women booking routine antenatal care. All women with a singleton pregnancy, attending for routine first-trimester nuchal translucency ultrasound assessment, were offered the option to participate in the study. Written informed consent was obtained from them, and the study was approved by the local ethics committee.

Transabdominal UtAD assessment was performed by the sonographer at the time of the nuchal translucency scan. UtAD indexes were measured as described previously. ${ }^{16}$ In brief, the paracervical vascular plexus was identified and color Doppler was used to identify the uterine artery as it made its ascent to the uterine body. Pulsed-wave Doppler was used to obtain uterine artery waveforms. When three similar consecutive waveforms were obtained, the presence of a protodiastolic notch was recorded and the resistance index (RI) was measured. The RI was preferred to the pulsatility index (PI) in this study because it has demonstrably better intraobserver and interobserver measurement repeatability. ${ }^{19}$ The patients and their clinicians were blinded to the results of the first-trimester UtAD assessment. Patient characteristics, including demographic details, and obstetric and medical histories were obtained at the first hospital visit and entered into our database. All pregnancy outcomes were obtained from the delivery suite database. Cases with fetal chromosomal or structural abnormalities, intrauterine infection, toxic insult (alcohol or drugs), medication (aspirin, heparin, antioxidants, or steroids), or concurrent maternal disease (eg, renal disease, connective tissue disease, malnutrition, cardiac disease, and diabetes) were excluded from the study. Patients who had a previous pregnancy affected by PE were included in the study; however, according to the exclusion criteria, none was routinely prescribed prophylactic therapy.

FGR was defined as a birth weight $<10^{\text {th }}$ percentile for gestational age (GA) with abnormal Doppler indexes (umbilical artery PI $>95^{\text {th }}$ percentile, middle cerebral artery $\mathrm{PI}<5$ th percentile, or ductus venosus PI $>95^{\text {th }}$ percentile for GA). Stillbirth was defined as intrauterine demise after 24 weeks' gestation. PE was defined after 20 weeks' gestation, according to the guidelines of the International Society for the Study of Hypertension in Pregnancy. This requires two recordings of diastolic blood pressure of $90 \mathrm{mmHg}$ at least 4 hours apart in previously normotensive women, proteinuria of $300 \mathrm{mg}$ or more in 24 hours, or two readings of at least $2+$ on dipstick analysis of midstream or catheter urine specimens if no 24-hour collection is available. ${ }^{20}$ Pregnancy outcomes from this data set have previously been published in several prospective series. ${ }^{13,14,21-24}$ However, the results were analyzed with an emphasis on screening performance rather than the positive predictive value for placental complications; we, therefore, present the data with these results.

\section{Doppler Ultrasound Characterization and Tissue Collection}

Determination of uterine artery RI was performed in women attending a clinic for termination of pregnancy in the first trimester, as previously described, ${ }^{16}$ at St. George's Hospital (London, UK). Ethical committee approval and full written consent were obtained (reference, 01.96 .8 and 01.78.5). Inclusion criteria were singleton pregnancy, GA of 9 to 14 weeks by crown-rump length (assigned by transvaginal measurement in accordance with local unit clinical policy), normal fetal anatomy, and nuchal translucency with no known maternal medical condition or history of recurrent miscarriage. High-resistance cases were defined as a mean RI $>95$ th percentile with bilateral diastolic notches. Normal-resistance cases had a mean RI of $<95$ th percentile. Tissue obtained from first-trimester surgical terminations of pregnancy was collected and rinsed in ice-cold phosphate-buffered saline. Placental villous tissue was separated from the decidua by blunt dissection and was randomly sampled and divided. Approximately $100 \mathrm{mg}$ of tissue was snap frozen in liquid nitrogen in two to three aliquots and stored at $-70^{\circ} \mathrm{C}$ until use.

\section{Protein Extraction}

Placental villous tissue (approximately $100 \mathrm{mg}$ ) was placed in a lysing tube (Matrix D; MP Biomedicals, Santa Ana, 
CA) with $1 \mathrm{~mL}$ ice-cold radioimmunoprecipitation assay buffer with protease inhibitors $(1 \mathrm{mmol} / \mathrm{L}$ phenylmethylsulfonyl fluoride, $60 \mu \mathrm{g} / \mathrm{mL}$ aprotinin, and 1 $\mathrm{mmol} / \mathrm{L}$ sodium orthovanadate). This was then homogenized (Fast Prep 24; MP Biomed) for two cycles of 30 seconds. The extract was then incubated on ice for 20 minutes and then centrifuged at $4^{\circ} \mathrm{C}$ at $15,000 \times g$ for 15 minutes. The supernatant was then further centrifuged at $4^{\circ} \mathrm{C}$ at $10,000 \times g$ for 10 minutes. Protein estimation was performed on the supernatant by Bradford assay (SigmaAldrich, St. Louis, MO).

\section{Western Blot Analysis}

Placental lysates were prepared in Laemmli buffer [50 $\mathrm{mmol} / \mathrm{L}$ Tris- $\mathrm{HCl}(\mathrm{pH} 6.8), 2 \%(\mathrm{w} / \mathrm{v})$ SDS, $10 \%(\mathrm{v} / \mathrm{v})$ glycerol] at $1 \mathrm{mg} / \mathrm{mL}$ with $3 \%$ (w/v) $\beta$ 2-mercaptoethanol and heated to $90^{\circ} \mathrm{C}$ for 5 minutes. Proteins were separated by SDS-PAGE and transferred to a polyvinylidene difluoride membrane (Hybond-P; Amersham, Buckinghamshire, UK), and Western blot analysis was performed, as previously described, ${ }^{25}$ using 1:1000 rabbit anti-BAX (catalog number 2772; Cell Signaling, Danvers, MA), 1:1000 rabbit anti-BCl2 (2872; Cell Signaling), 1:1000 mouse anticaspase 3 (9668; Cell Signaling), 1:5000 rabbit anticleaved poly (ADP-ribose) polymerase (G7341; Promega, Fitchburg, WI), 1:1000 rabbit anti-heat shock protein 70 (SPA 812; Enzo Life Sciences, Exeter, UK), 1:1000 mouse anti-heme-oxygenase 1 (ab13248; Abcam, Cambridge, UK), 1:1000 mouse anti-hypoxia-inducible factor (HIF) $1 \alpha$ (610958; BD Transduction, BD Biosciences, Oxford, UK), 1:1000 rabbit anti-HIF2 $\alpha$ (NB100-480; Novus Biologicals, Littleton, CO), 1:1000 mouse anti-nitrotyrosine (ab7048; Abcam, Cambridge, UK), 1:5000 mouse anti-insulin-like growth factor (IGF)-1 (ab40789; Abcam), 1:1000 mouse anti-IGF2 (ab63294; Abcam), 1:10,000 rabbit anti-actin (ab2066; Abcam), and 1:10,000 mouse anti- $\alpha$-tubulin (ab7291; Abcam). The blots were scanned and densitometry was performed using ImageJ software version $1.43 \mathrm{u}$ (NIH, Bethesda, MD). Results are expressed as a ratio of protein of interest/loading control (actin or tubulin). Nitrotyrosine residues are detected on a large range of proteins of different molecular weights; therefore, a slot blot matrix was used to load $20 \mu \mathrm{g}$ protein in phosphatebuffered saline directly onto two polyvinylidene difluoride membranes and left to air dry for 1 hour at room temperature. Treatment of the membranes thereafter was as described above.

\section{Apoptosis Array}

A human apoptosis antibody array (AAH-APO; Ray Biotech, Norcross, GA) was incubated for 12 hours with pooled high RI and normal RI lysates $(n=8$ in each group, total of $600 \mu \mathrm{g}$ of protein, $75 \mu \mathrm{g}$ from each individual placental lysate), as per the manufacturer's instructions.
Antioxidant Enzyme Activity and Lipid Peroxidation Assay

Glutathione peroxidase assay (703102; Cayman Chemicals, Ann Arbor, MI), superoxide dismutase (SOD) assay (706002; Cayman Chemicals), and lipid peroxidation assay (437634; Calbiochem, Merck Millipore, Billerica, MA) were performed on 10 to $20 \mu \mathrm{L}$ of placental lysate, prepared per the manufacturer's instructions. Final enzyme activity was calculated by correcting for protein concentration, determined by Bradford assay.

\section{Detection of VEGF-A and Leptin}

Total vascular endothelial growth factor (VEGF)-A and leptin were detected in placental lysates using enzyme-linked immunosorbent assay [ELISA; Leptin DY398 Human DuoSet (R\&D Systems Europe, Abingdon, UK) and VEGF-A ELISA 900-K10 (Peprotech, London, UK)], according to the manufacturer's instructions. Sample concentrations were interpolated from the standard curves and adjusted for protein concentration, determined by Bradford assay.

\section{Immunohistochemistry}

Placental tissue was formalin fixed and paraffin embedded, and sections (10 $\mu \mathrm{m}$ thick) were cut. Slides were dewaxed in xylene for 10 minutes, followed by rehydration through graded ethanol $(100 \% \mathrm{w} / \mathrm{v}, 95 \%$ $\mathrm{w} / \mathrm{v}, 80 \% \mathrm{w} / \mathrm{v}$, and $70 \% \mathrm{w} / \mathrm{v}$ ) for 5 minutes each, with a final wash in water. Antigen retrieval was performed in boiling tris-HCL buffer (10 mmol/L, pH 10) for 10 minutes. Sections were then washed in tris-buffered saline (TBS), permeabilized in TBS $/ 0.2 \%$ (v/v) Triton X-100 (SigmaAldrich) for 5 minutes, and again washed in TBS. They were then blocked in TBS $/ 10 \%(\mathrm{v} / \mathrm{v})$ goat serum $/ 1 \%(\mathrm{w} / \mathrm{v})$ bovine serum albumin for 1 hour at room temperature and again washed with TBS. Mouse anti-human cytokeratin M30 (0.5 $\mu \mathrm{g} / \mathrm{mL})$ or cytokeratin $7(0.62 \mu \mathrm{g} / \mathrm{mL})$ was then applied and incubated at $4^{\circ} \mathrm{C}$ overnight. Control sections were included with nonimmune $\mathrm{IgG}(0.5$ or $0.62 \mu \mathrm{g} / \mathrm{mL})$. After further washes in TBS, the biotinylated and horseradish peroxidaseconjugated secondary antibody was applied for 10 minutes at room temperature (Histofine kit; Invitrogen, ThermoFisher Scientific, Paisley, UK). Sections were incubated with diaminobenzidine (Dako, Ely, UK) for 1 to 5 minutes until positive staining was identified by a brown color under microscopy. Sections were then mounted and visualized, and digital images were stored [IX70 inverted microscope (Olympus, Southend-on-Sea, UK) and C4742-95 digital camera (Hamamatsu Photonics, Welwyn Garden City, UK)].

\section{TUNEL Staining}

Sections were prepared as above, then incubated in permeabilization solution $[0.1 \%(\mathrm{v} / \mathrm{v})$ Triton $\mathrm{X}-100$ and $0.1 \%$ 
(w/v) sodium citrate] for 8 minutes. Terminal deoxynucleotidyl transferase-mediated dUTP nick-end labeling (TUNEL) staining was performed per manufacturer's instructions (catalog number 11767305001; Roche, Welwyn Garden City, UK). Sections were visualized by fluorescence microscopy, and digital images were captured as previously described in Immunohistochemistry.

\section{Microarray Analysis}

Placental samples from GA-matched pregnancies in high RI and normal RI cases $(n=11)$ were collected as above. Total mRNA was isolated with a combined protocol of QIAzol lysis reagent and Qiagen RNeasy mini kit (including the RNaseFree DNase set; Qiagen, Manchester, UK), according to the manufacturer's protocol. RNA quantity and quality were confirmed by RNA 6000 Chip in the 2100 Bioanalyzer (Agilent Technologies, Santa Clara, CA) and NanaDrop UV/ VISSpectrometer (PeqLab, VWR, Lutterworth, UK). Microarray studies were performed with the Human HT12_V3_0_R2 (Illumina, San Diego, CA), according to the minimum information about a microarray experiment criteria. Briefly, total RNAs were hybridized to the Illumina array after labeling using the Illumina TotalPrep RNA amplification kit. Illumina GenomeStudio software version 2011.1 with gene expression module version 1.9.0 was used to export probe-level data without normalization or background correction. For analysis, Idat files were imported to GenomeStudio (Illumina), and raw data were exported in text format. Data were analyzed in Gene Spring version 11.5.1 (Agilent Technologies). In brief, data were normalized using quantile normalization and baseline transformed to the median of all control samples. Sample distributions were checked for consistency and conformity to a gaussian distribution by qualitative methods, and replicate consistency was assessed using principal component analysis. Data were filtered to remove unexpressed or unreliable data such that remaining entities should have a detection $P>0.6$ in $100 \%$ of samples for any one of the two conditions. The most differentially regulated genes were identified by using a fold change of 1.5 filter and $t$-test with a corrected $P<0.05$. Gene ontology analysis was performed using a corrected $P<0.1$ to allow a larger gene set for analysis. Gene ontology categories relating to a biological process were determined by Ariadne pathway studio analysis (Elsevier, London, UK) and by AmiGO 2 database entry for each gene (http://amigo. geneontology.org/amigo, last accessed June 1, 2015). ${ }^{26}$ Microarray data have been submitted to the European Bioinformatics Institute Array Express data repository (http://www. ebi.ac.uk/arrayexpress, accession number E-MTAB-3265).

\section{Statistical Analysis}

Data were analyzed with GraphPad Prism version 6 (GraphPad, San Diego, CA). Normal distribution was assessed by Kolmogorov-Smirnov tests. Groups were compared by using a Student's unpaired $t$-test and are presented as means \pm SEM.

\section{Results}

Outcomes in Ongoing Pregnancies with First-Trimester High-Resistance Uterine Artery Blood Flow

In our data set of 9952 ongoing pregnancies, cases with UtAD RI $>95^{\text {th }}$ percentile in the first trimester $(n=568)$ had a $15 \%$ risk of PE ( 86 of 568) compared with a $2.8 \%$ risk in cases with UtAD RI $<95$ th percentile (264 of 9384). In all, women with the highest degree of placental resistance had a $24 \%$ chance of developing a placental complication of pregnancy (PE, FGR, or stillbirth) compared with women $<95^{\text {th }}$ percentile who had a $4.9 \%$ risk. Full demographic details are listed in Supplemental Table S1. Pregnancy outcomes from this data set have previously been published in several prospective series $^{13,14,21,23,27}$; however, the results were analyzed with an emphasis on screening performance rather than the positive predictive value for placental complications.

\section{Gene Expression Is Altered in Placental Tissue from High RI Pregnancies}

Placental gene expression has been shown to be altered at the time of delivery in PE and FGR, particularly in pathways involving inflammation, oxidative stress, and hypoxia. ${ }^{28,29}$ There is a paucity of data in pregnancies with poor placentation in the first trimester, with two studies from chorionic villous sampling reporting altered gene expression months before the onset of clinical disease. ${ }^{30,31} \mathrm{We}$, therefore, determined placental gene expression by microarray in GAmatched high RI and normal RI cases. We found that 26 genes were significantly differentially expressed in the high RI versus the normal RI group ( $>1.5$-fold change, $P<0.05$ ); six of these demonstrated up-regulation of expression, whereas the remaining 23 were down-regulated. The genes and their gene ontology are presented in Table 1. Gene ontology analysis identified seven ontologies overrepresented in the biological process category; these included immune system response, cell death and apoptosis, response to stress, cyclooxygenase pathway, inflammatory response, and metabolics. Pathways involving hypoxia and oxidative stress did not appear altered (Supplemental Figure S1).

\section{HIF1 $\alpha$ Protein Expression Is Reduced in Placental Tissue from High RI Pregnancies}

HIF $1 \alpha$ and HIF $2 \alpha$ are master regulators of the hypoxia response $^{32}$ and are known to be expressed in the firsttrimester placenta. ${ }^{33}$ Placental hypoxia has been implicated in the pathogenesis of PE, both at the time of disease and in the first trimester, ${ }^{33,34}$ and increased HIF expression in placenta from pregnancies with $\mathrm{PE}$ has been demonstrated. ${ }^{35}$ We evaluated protein expression of HIF $1 \alpha$ and HIF $2 \alpha$ in high RI and normal RI placental tissues. Significantly lower levels of HIF1 $\alpha$ were found $(P=0.022, n=17$ and 16 , respectively) (Figure 1A); however, HIF2 $\alpha$ levels were not 
Table 1 Differentially Expressed Genes in High RI Compared with Normal RI Placental Tissue

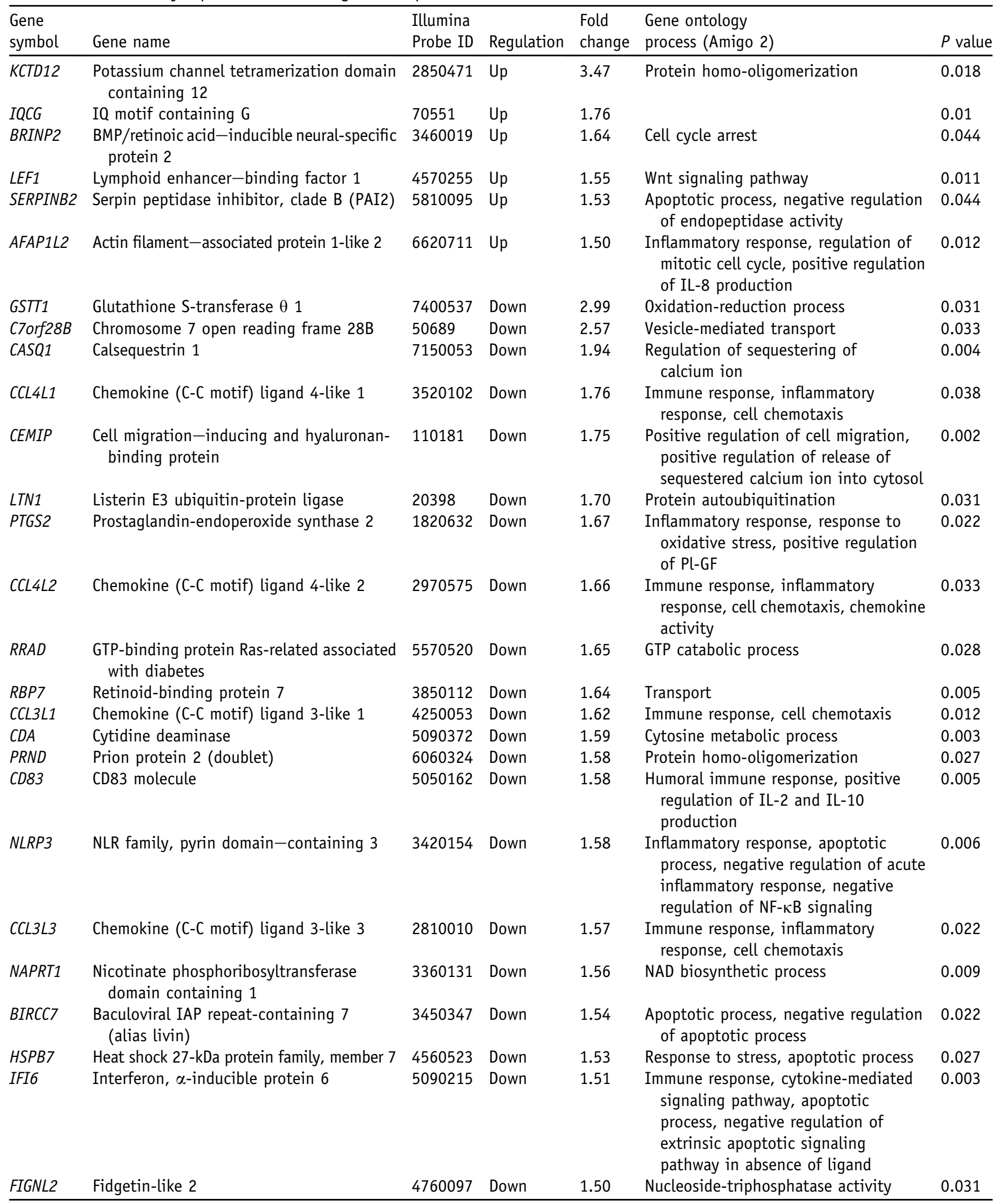

Illumina microarray of gestational age (GA)-matched, first-trimester placental tissue $(n=11)$. GA (in days) for normal, 73.64 \pm 5.1 ; high, 73.82 \pm 3.9 . Gene ontology categories relating to a biological process determined by Ariadne pathway studio analysis and by AmiG0 2 database entry for each gene. ${ }^{26}$

BMP, bone morphogenetic protein; IAP, inhibitor of apoptosis; NLR, nucleotide-binding domain and leucine-rich repeat containing; PAI, plasminogen activator inhibitor; Pl-GF, placental growth factor; RI, resistance index. 
different $(P=0.553, n=16$ and 15 , respectively $)$ (Figure 1B).

VEGF-A is a known important downstream target of HIF signaling, is abundant in the first-trimester placenta, and has important roles in regulating angiogenesis. ${ }^{36}$ Leptin has been shown to be dysregulated in $\mathrm{PE}^{29}$ and is also a target of HIF signaling. We, therefore, determined the levels of these proteins by ELISA in placental lysate. High RI and normal RI pregnancies did not differ in the level of either total VEGF-A or leptin ( $n=16$ in each group) (Figure 1, C and $\mathrm{D}$, respectively).

\section{Placental Tissue in the First Trimester Demonstrates Evidence of Oxidative Stress}

Mild oxidative stress occurs in normal pregnancy, ${ }^{37}$ and a burst of oxidative stress occurs at 10 to 12 weeks' gestation as the fetoplacental unit adapts to an increase in oxygen. ${ }^{38}$ The evidence linking oxidative stress and PE is convincing ${ }^{39}$; however, the mechanisms of pathogenesis and the timing of the onset of oxidative stress are less well understood. We evaluated several different end points of oxidative stress within placental tissue, including nitrotyrosine residues, lipid peroxidation, and heat shock protein 70 levels. There was evidence of oxidative stress in all samples tested; however, no difference in the level of placental tissue markers of oxidative stress was found in pregnancies with high RIs compared with normal indices (Figure 2, A-C).

\section{GPx Activity Decreases and SOD Activity Increases in Placental Tissue from High RI Pregnancies}

The expression and activity of antioxidant enzymes increase at 10 to 12 weeks' gestation, concomitant with the onset of maternal perfusion of the placenta ${ }^{38}$; aberrant antioxidant defenses at the time of disease have been implicated in the pathogenesis of $\mathrm{PE}^{40,41} \mathrm{We}$ assessed enzyme activity of glutathione peroxidase (GPx) and SOD with the aim of quantifying the functional level of these two important antioxidant systems within the placenta. Significantly lower GPx activity was seen in the placental tissue from high RI pregnancies ( $P=0.007, n=10$ in each group) (Figure 2D). In contrast, higher SOD activity was demonstrated in the high RI group $(P=0.014, n=8$ and 9 in two groups) (Figure 2E).

\section{Apoptosis Increases and IGF2 Protein Expression Decreases in Placental Tissue from High RI Pregnancies}

We have previously shown that first-trimester extravillous trophoblasts from high RI pregnancies are more sensitive to apoptotic stimuli, ${ }^{17}$ and higher levels of placental apoptosis have been shown in pregnancies with PE and FGR. ${ }^{9,10} \mathrm{We}$, therefore, investigated markers of placental apoptosis in high RI and normal RI placentas by Western blot analysis. High RI cases had significantly higher levels of cleaved caspase $3(P=0.0002, n=16$ in each group) (Figure 3A)

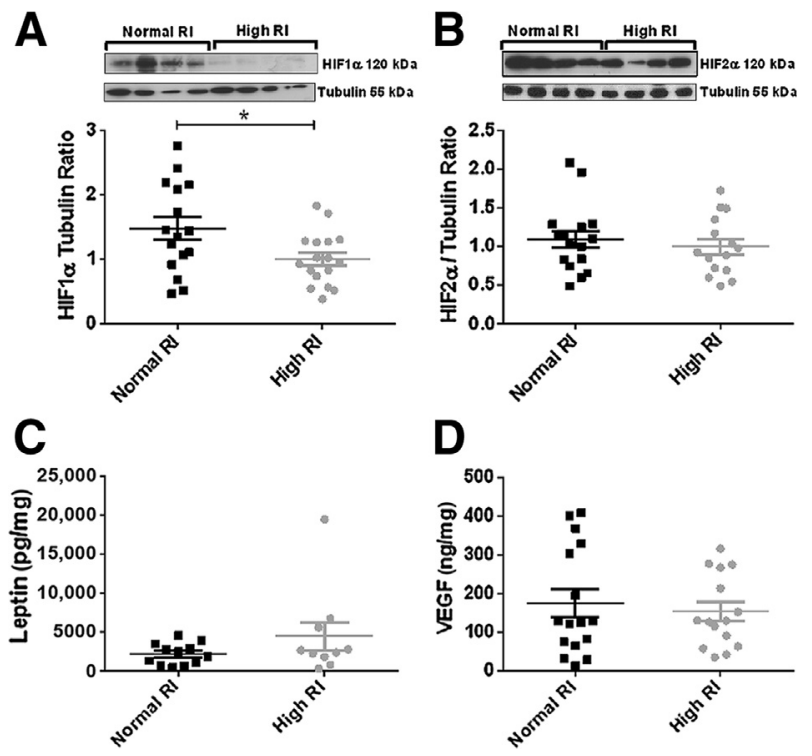

Figure 1 Hypoxia-inducible factor (HIF), total vascular endothelial growth factor (VEGF)-A, and leptin protein expression in placental lysate from high resistance index (RI) and normal RI pregnancies. A: HIF1 $\alpha$ expression determined by Western blot analysis of high RI and normal RI placental lysate for HIF1 $\alpha(120 \mathrm{kDa})$, with tubulin $(55 \mathrm{kDa})$ detected as loading control, is significantly lower in high RI cases $(P=0.022)$. Gestational age (GA; in days) for normal, $79.44 \pm 2.5$; and high, $76.25 \pm 3.5$. B: HIF2 $\alpha$ expression determined by Western blot analysis of high RI and normal RI placental lysate for HIF2 $\alpha$ (120 kDa), with tubulin $(55 \mathrm{kDa})$ detected as loading control, is not different between the two groups $(P=0.553)$. GA (in days) for normal, $75.06 \pm 2.5$; high, $82.81 \pm 2.7$. C: Leptin protein expression, determined by enzyme-linked immunosorbent assay (ELISA) in high RI and normal RI placental lysate and expressed as $\mathrm{pg} / \mathrm{mg}$ protein concentration, shows no significant difference $(P=0.186)$. GA (in days) for normal, $82.81 \pm 2.7$; high, $74.54 \pm 3.07$. D: Total VEGF-A protein expression determined by ELISA and expressed as $\mathrm{ng} / \mathrm{mg}$ protein concentration, shows no significant difference $(P=0.606)$. GA (in days) for normal, $79.62 \pm 2.5$; high, $73.46 \pm 2.7$. Scatter plot: each point represents an individual placental sample. Data are presented as means \pm SEM (statistical analysis $t$-test). $n=$ 16 (A, B, and D, high RI); $n=17$ (A, normal RI); $n=15$ (B and D, normal $\mathrm{RI}) ; n=12$ (C, high RI); $n=10$ (C, normal RI). ${ }^{*} P<0.05$.

and cleaved poly (ADP-ribose) polymerase $(P=0.0167$, $n=16$ in each group) (Figure $3 \mathrm{~B}$ ).

Immunohistochemical analysis confirmed apoptosis by TUNEL and CkM30 staining (Figure 4, A-F). We performed a protein array on pooled lysate $(n=8$ in each group) (Supplemental Table S2) to provide candidates for further investigation of apoptotic pathways. The array data suggested down-regulation of IGF2; therefore, we investigated it in placental lysates from individual patients. Significantly lower levels of IGF2 were detected in high RI pregnancies ( $P=0.04, n=16$ in each group) (Figure 5B); however, no significant differences in IGF1 were seen $(n=$ 16 in each group) (Figure 5A). Placental studies in PE and FGR have suggested alterations in intrinsic mitochondrial regulation of apoptosis. ${ }^{42} \mathrm{We}$, therefore, investigated placental Bax and Bcl-2 expression (proapoptotic and antiapoptotic, respectively), with no difference seen between high RI and normal RI tissues (Bax, $n=11$ and 12; Bcl-2, $n=16$ in each group) (Figure 5, C and D). 

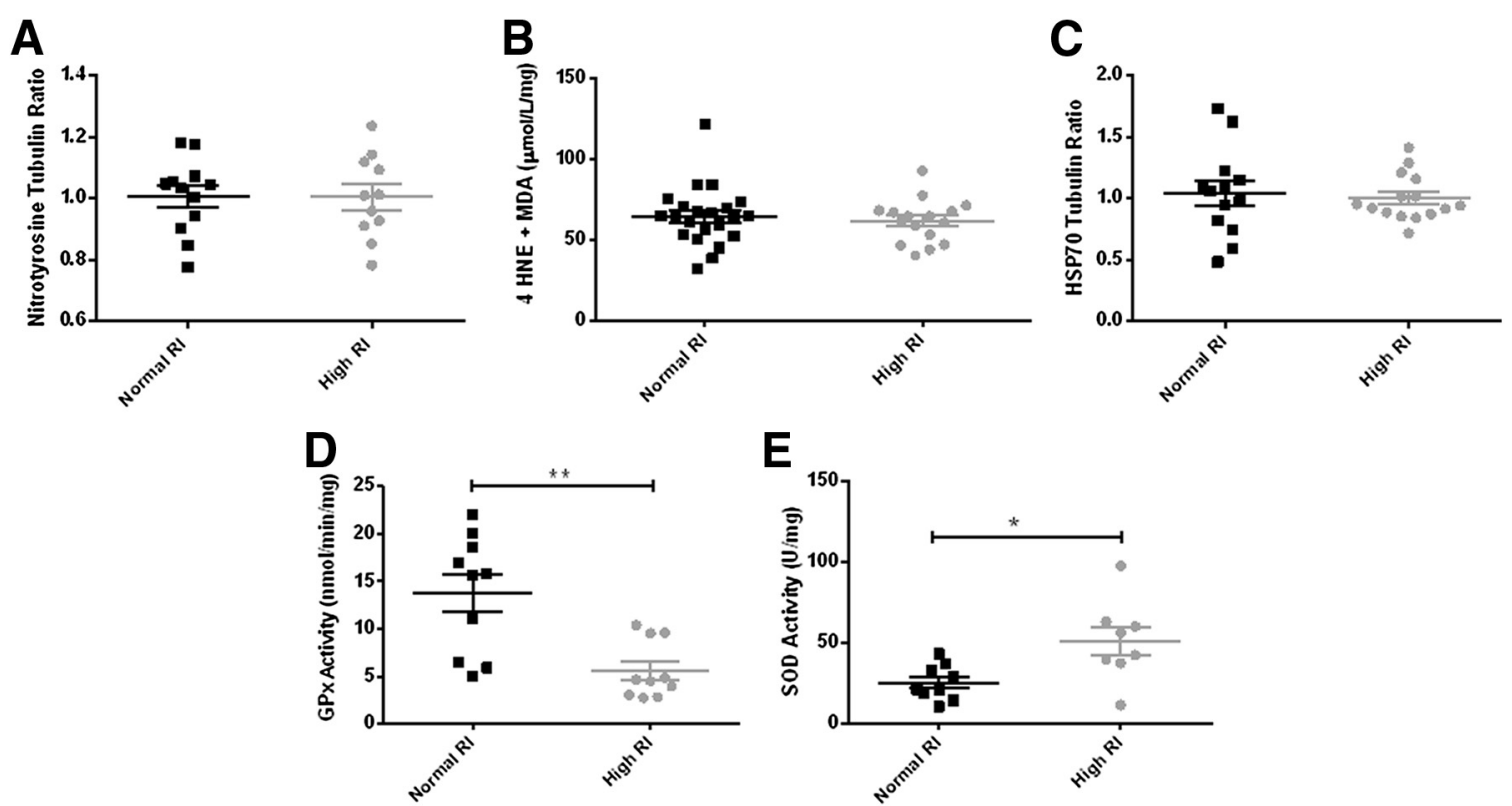

Figure 2 Expression of markers of oxidative stress and antioxidant enzyme activity in placental lysate from high resistance index (RI) and normal RI pregnancies. A: Nitrotyrosine expression. Nitrotyrosine/tubulin ratio by densitometry, immunoblotting of high RI, and normal RI placental lysate loaded onto polyvinylidene difluoride (PVDF) membrane by slot blot matrix, shows no difference ( $P=0.95)$. Gestational age (GA; in days) for normal, $74.75 \pm 2.9 ;$ high, $80.67 \pm 3.12$. B: Lipid peroxidation measured by 4-hydroxynonenal (4 HNE) and malondiadehyde (MDA) assay, shows no difference $(P=0.59)$. C: Heat shock protein (HSP) 70 expression determined by Western blot analysis of high RI and normal RI placental lysate for HSP 70 (70 kDa), with tubulin (55 kDa) detected as loading control, shows no difference $(P=0.689)$. GA (in days) for normal, $75.81 \pm 2.7$; high, $78.50 \pm 2.6$. D: Glutathione peroxidase (GPX) activity measured by assay (703102; Cayman Chemicals). GPx enzyme activity significantly reduces in high RI cases ( $P=0.007)$. GA (in days) for normal, $80.8 \pm 3.3$; high, $76.3 \pm 3.2$. E: Superoxide dismutase (SOD) activity measured by assay (number 706002; Cayman Chemicals). S0D enzyme activity is higher in high RI cases $(P=0.014)$. GA (in days) for normal, $81.3 \pm 2.6$; high, $78 \pm 3.2$. Scatter plots: each point represents an individual placental sample. Data are presented as means \pm SEM (statistical analysis t-test). $n=12$ (A, high RI); $n=11$ (A, normal RI); $n=22$ (B, high RI); $n=16(\mathbf{B}$, normal RI); $n=13(\mathbf{C}$, high RI); $n=15$ (C, normal RI); $n=10$ (D, high and normal RI); $n=8$ (E, high RI); $n=9$ (E, normal RI). *P<0.05, **P<0.01.

\section{Discussion}

Investigating the pathogenesis of the clinical consequences of poor placentation, such as FGR, PE, and stillbirth, has traditionally been hampered by our inability to study these pregnancies in the first trimester when the critical events for successful placental development occur. Interventions have, to date, also been disappointing, with aspirin providing only modest benefit and delivery remaining the only cure. ${ }^{6}$ It is likely that future interventions will need to start early in pregnancy, long before the clinical consequences become evident, and a fundamental understanding of the mechanisms that determine both sufficient and insufficient placentation is required. ${ }^{43}$ We have used the ability to assess impedance to uterine artery flow in the first trimester to study those pregnancies with the highest resistance to flow in comparison to those with normal parameters.

\section{0xygen Signaling}

The first-trimester placenta is known to develop in a low oxygen environment, with oxygen levels of $20 \mathrm{mmHg}$ at 8 weeks' gestation, increasing to $>50 \mathrm{mmHg}$ at 12 weeks, concomitant with the onset of maternal arterial perfusion. ${ }^{38}$ The association between placental oxidative stress and PE in later pregnancy is well established, ${ }^{40,44}$ although it remains unclear whether hypoxia or reperfusion is causal of this, with a paucity of in vivo data on placental oxygenation in complicated pregnancy. Early hypoxia in the first-trimester placenta has been implicated in the pathogenesis of $\mathrm{PE}$ and placental insufficiency, ${ }^{33}$ although this theory and a causal role for placental hypoxia have been challenged. ${ }^{4,45}$ There was no evidence in our gene expression data of an up-regulation of hypoxia or oxidative stress genes in placental tissue from pregnancies with high-resistance flow. Consistent with our findings, two previous studies using stored placental samples from chorionic villous sampling of women who subsequently developed PE have also demonstrated no evidence of an increased hypoxic or oxidative stress response in first-trimester placental gene expression. ${ }^{30,31}$

HIF $1 \alpha$ is broken down rapidly in conditions of normoxia, and its levels are directly related to the degree of cellular hypoxia. ${ }^{46}$ We found significantly lower levels of HIF1 $\alpha$ in high RI pregnancies that may be a direct reflection of higher levels of oxygen in vivo. This paradoxically higher level of oxygen may result from deficient plugging of the spiral arteries by extravillous trophoblast and premature perfusion of the placenta by maternal blood, a process that has also been implicated in early pregnancy failure. ${ }^{47}$ Oxygen-independent regulation is also important; inflammatory mediators, cytokines, growth factors, ${ }^{48}$ and the renin-angiotensin system ${ }^{49}$ have been reported to regulate HIF1 $\alpha$ and are abundant in 

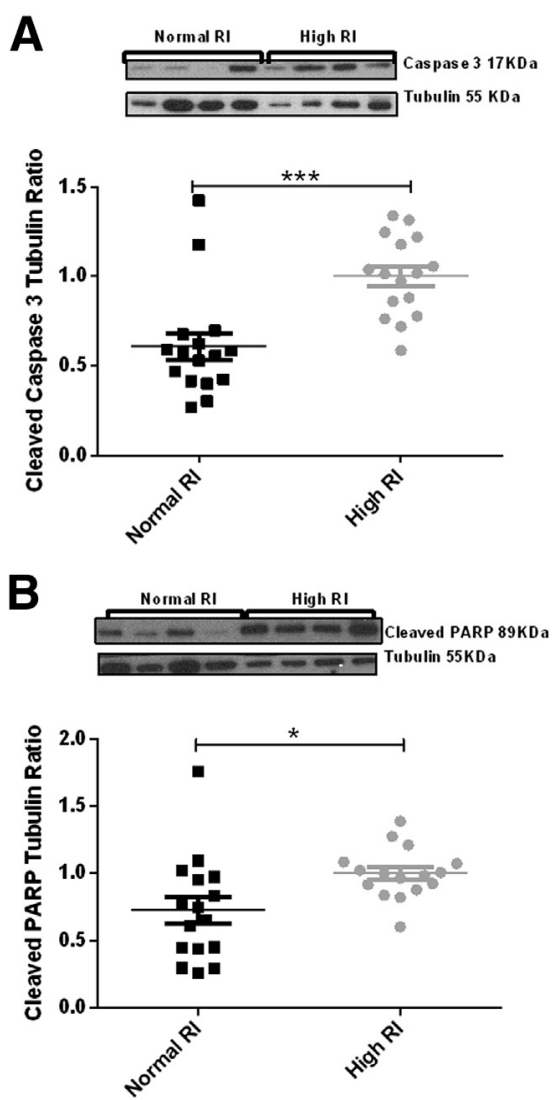

Figure 3 Apoptotic markers in placental lysate from high resistance index (RI) and normal RI pregnancies. A: Caspase 3 protein expression determined by Western blot analysis of high RI and normal RI placental lysate for caspase 3 $(17 \mathrm{kDa})$, with tubulin $(55 \mathrm{kDa})$ detected as loading control, significantly increases in high RI placental tissue $(P=0.0002)$. Gestational age $(\mathrm{GA}$; in days) for normal, $79.3 \pm 2.3$; high, $74.8 \pm 2.6$. Representative image. B: Cleaved poly (ADP-ribose) polymerase (PARP) protein expression determined by Western blot analysis of high RI and normal RI placental lysate for cleaved PARP ( $89 \mathrm{kDa}$ ), with tubulin ( $55 \mathrm{kDa}$ ) detected as loading control, significantly increases in high RI pregnancies ( $P=0.0167)$. GA (in days) for normal, $80.25 \pm 2.5 ;$ high, $75.3 \pm 2.7$. Representative image. Scatter plot: each point represents an individual placental sample. Data are presented as means \pm SEM (statistical analysis $t$-test). $n=16$ in each group (A and $\mathbf{B}$ ). ${ }^{*} P<0.05$, $* * * P<0.0005$.

the first-trimester placenta. ${ }^{50}$ Our microarray data provide evidence of an altered inflammatory environment in high RI pregnancies with the most differentially regulated genes, including PTGS2 (cyclooxygenase 2), and several CCL3 and CCLA chemokines.

Lower levels of HIF1 $\alpha$ may represent a response to an aberrant placental inflammatory environment, and further investigation of these inflammatory markers and of the activation state of fetal villous macrophages would be of interest. We have also shown aberrant chemokine and cytokine production by decidual natural killer cells from high RI pregnancies, suggesting that alterations in the inflammatory microenvironment may extend across the maternal-fetal interface in high RI pregnancies. ${ }^{18,51}$

We observed no difference in HIF $2 \alpha$ levels, suggesting that mechanisms specific to HIF1 $\alpha$ may be involved. Although the localization and temporal expression of both HIF1 $\alpha$ and HIF2 $\alpha$ have been studied in the human placenta, the complexity of their regulation remains poorly understood in comparison to their behavior in other tissue types and human cancer. ${ }^{50,52}$ Alterations in the relative balance between HIF1 $\alpha$ and HIF $2 \alpha$ in other human tissue have been reported to have effects on cell proliferation, invasion, and angiogenesis, processes also important in placentation. ${ }^{53}$ The functional effects in the human placenta remain to be elucidated.

IGF2, which is a known HIF1 $\alpha$-specific target, was down-regulated at a protein level; however, total VEGF-A and leptin were not. HIF $1 \alpha$ and HIF $2 \alpha$ are known to act on $>100$ target genes via the hypoxia response element, and redundancy in signaling may reduce a downstream effect ${ }^{54}$; in addition, the expression of these proteins is controlled by many intersecting pathways beyond the HIF pathway alone. Villous tissue is a complex mix of several trophoblast types, fetal stromal cells, macrophages, and endothelial cells. Absence of changes in a particular pathway could simply be because of a dilutional effect or heterogeneous expression.

\section{0xidative Stress and Antioxidant Defenses}

There was evidence of oxidative stress in all first-trimester placental tissue studied using a variety of tissue markers;
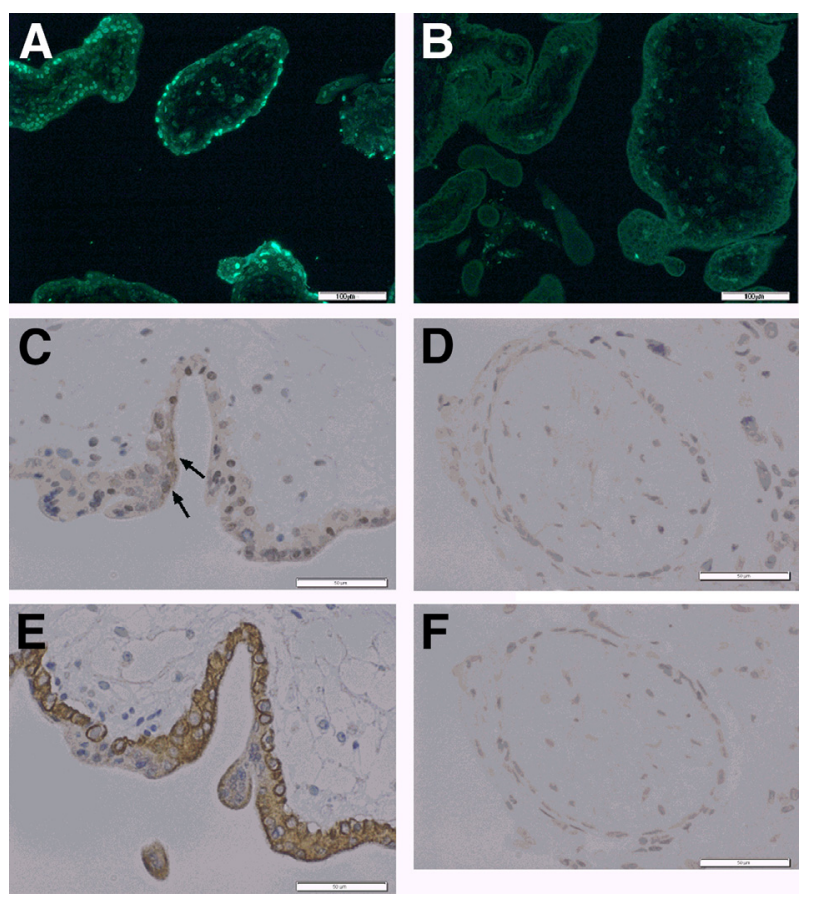

Figure 4 Terminal deoxynucleotidyl transferase-mediated dUTP nickend labeling (TUNEL) and cytokeratin M30 immunohistochemistry (IHC) of first-trimester placental tissue. IHC confirming apoptosis [high resistance index (RI), with a gestational age of 72 days]. A: TUNEL fluorescence. B: Negative control TUNEL assay. C: Cytokeratin M30 staining. D: IgG control for CkM30. E: Cytokeratin 7 staining of a serial section. F: IgG control for $\mathrm{Ck} 7$. Arrows demonstrating $\mathrm{CkM} 30$ staining apparently localizing in the syncytiotrophoblast cytoplasm. Scale bars: $100 \mu \mathrm{m}$ (A and B); $50 \mu \mathrm{m}$ $(\mathbf{C}-\mathbf{F})$. 

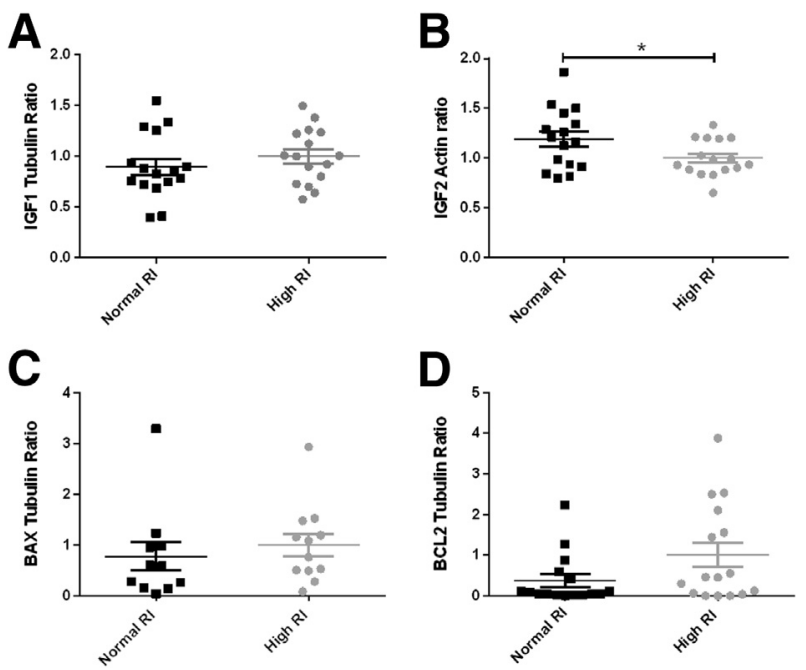

Figure 5 Expression of regulators of apoptosis in placental lysate from high resistance index (RI) and normal RI pregnancies. A: Insulin-like growth factor (IGF)-1 protein expression determined by Western blot analysis of high RI and normal RI placental lysate for IGF1 (20 kDa), with tubulin $(55 \mathrm{kDa})$ detected as loading control, is not significantly different $(P=0.355)$. Gestational age (GA; in days) for normal, $79.31 \pm 2.3$; high, $74.33 \pm 2.6$. B: IGF2 protein expression, determined by Western blot analysis for IGF2 $(50 \mathrm{kDa})$ with actin $(36 \mathrm{kDa})$, detected as loading control, is significantly reduced in high RI cases $(P=0.040)$. GA (in days) for normal, $80.94 \pm 2.4$; high, $77.13 \pm 2.5$. C: Bax protein expression, determined by Western blot analysis for Bax (20 kDa), with tubulin (55 kDa) detected as loading control. Bax was not significantly different $(P=0.5287)$. GA (in days) for normal, $75.81 \pm 2.7$; high, $78.50 \pm 2.6$. D: $\mathrm{Bcl}-2$ protein expression, determined by Western blot analysis for Bcl-2 (28 $\mathrm{kDa})$, with tubulin $(55 \mathrm{kDa})$ detected as loading control. Bcl-2 is not significantly different between two groups ( $P=0.0722)$. GA (in days) for normal, $80.25 \pm 2.5$; high, $75.3 \pm 2.7$. Scatter plot: each point represents an individual patient sample. Data are presented as means \pm SEM (statistical analysis $t$-test). $n=16$ (A, B, and $\mathbf{D}$, high and normal RI); $n=11$ (C, high RI); $n=12$ (C, normal RI). ${ }^{*} P<0.05$.

however, placental tissues from high RI and normal RI pregnancies did not differ in their level of oxidative stress markers. Antioxidant defense systems become active in the first trimester, with increasing levels of GPx and SOD correlated with both GA and intervillous oxygen levels. ${ }^{38}$ High RI placental lysate had significantly lower levels of GPx activity and higher levels of SOD activity. An imbalance in placental antioxidant enzymes may ultimately result in oxidative stress in later pregnancy, even though no effect was seen in the first trimester. PE has been associated with reduced antioxidant enzyme activity. ${ }^{40,41}$ Other human tissue inflammatory mediators, in particular tumor necrosis factor $\alpha$, have been reported to up-regulate SOD activity. ${ }^{55}$ SOD activity in high RI cases may be up-regulated in response to deficient GPx activity. The underlying mechanisms leading to an altered antioxidant balance in the first trimester require further investigation.

\section{Apoptosis and IGF2}

Apoptosis is a highly regulated energy-dependent process that is initiated as a response to several different cell stresses, including hypoxia, oxidative stress, and inflammation. ${ }^{56}$ There is strong evidence to support increased placental apoptosis in obstetric complications of poor placentation, including PE and FGR. ${ }^{9,10}$ We found higher levels of apoptotic markers in the placenta from high RI pregnancies. We have previously demonstrated an increased sensitivity of primary trophoblasts from high RI pregnancies to apoptotic stimuli in vitro, and our findings confirm an in vivo effect. ${ }^{17}$ Trophoblasts from pregnancies affected by FGR or PE have also been shown to have increased sensitivity to apoptotic stimuli. ${ }^{57}$ Higher levels of apoptosis in the first trimester may lead to aberrant development of the villous architecture and fetal vasculature. It is known that fetuses affected by FGR have abnormal placental vessels and smaller placental mass, with the possible consequence of reduced nutrient and oxygen uptake. ${ }^{58}$

We found significantly lower levels of IGF2 in high RI placentas, which may potentially result in increased levels of apoptosis. In a first-trimester explant model, IGF2 has been shown to regulate the trophoblast cell cycle and protect against apoptosis. ${ }^{59}$ Apoptosis is considered to play a role in normal placental physiology, particularly in cytotrophoblast cell cycle control and the formation and maintenance of the integrity of the syncytiotrophoblast layer. ${ }^{60}$ IGF2 has effects on cell proliferation and invasion ${ }^{61}$; lower levels of IGF2 may conceivably reduce cell proliferation, placental mass, and trophoblast invasion in addition to increasing apoptosis. In the murine placenta, a regulatory effect of IGF2 has been demonstrated on HIF expression, with complex interactions between IGF2, oxygen, and HIF on murine trophoblast proliferation, migration, and invasion. However, this has not as yet been studied in the human placenta. ${ }^{48}$

It is not possible to determine the individual cell types affected from placental lysate; we, therefore, investigated this immunohistochemically by staining for TUNEL, cytokeratin M30, and cytokeratin 7, confirming apoptosis in the trophoblast, with apparent localization to the syncytiotrophoblast. Conflicting results have previously been published as to the site of apoptotic changes seen in both normal and pathological pregnancy, and more recent evidence highlights the difficulty of attributing cell lineage in the firsttrimester placenta with evidence that the cytotrophoblast may interdigitate into the syncytiotrophoblast layer. ${ }^{62}$

\section{Conclusions}

We provide evidence that first-trimester pregnancies with high RI have differentially regulated placental gene expression, higher placental levels of apoptosis, and lower levels of IGF2 compared with normal RI pregnancies. These changes are apparent several months before the clinical consequences of placental insufficiency become evident. Alterations in antioxidant defense systems and lower placental levels of HIF $1 \alpha$ are also seen, which may be because of oxygen-independent regulation as no evidence of differential hypoxia-related gene expression or exaggerated 
tissue oxidative stress was found in the first trimester. It is certainly possible that alterations in placental antioxidant balance may lead to increased placental oxidative stress in later pregnancy. Our data support the concept that in the first trimester, low oxygen levels and mild oxidative stress are a normal physiological state and may be important for many aspects of successful placental development. Neither hypoxia nor oxidative stress seem to be associated with poor placentation in the first trimester, and rather paradoxically high levels of oxygen or oxygen-independent regulation of HIF1 $\alpha$ may be important. The causal mechanisms for insufficient placentation in the first trimester and subsequent placental complications may involve increased apoptosis and deficiencies in IGF2. These pathways may potentially provide therapeutic targets to ameliorate or even prevent these potentially devastating placental complications.

\section{Acknowledgments}

We thank the clinic, theater, and Fetal Medicine Unit staff of St. George's Hospital for help with sample collection.

\section{Supplemental Data}

Supplemental material for this article can be found at http://dx.doi.org/10.1016/j.ajpath.2015.06.020.

\section{References}

1. Khong TY, De Wolf F, Robertson WB, Brosens I: Inadequate maternal vascular response to placentation in pregnancies complicated by pre-eclampsia and by small-for-gestational age infants. Br J Obstet Gynaecol 1986, 93:1049-1059

2. Brosens I, Pijnenborg R, Vercruysse L, Romero R: The "Great Obstetrical Syndromes" are associated with disorders of deep placentation. Am J Obstet Gynecol 2011, 204:193-201

3. Romero R, Kusanovic JP, Chaiworapongsa T, Hassan SS: Placental bed disorders in preterm labor, preterm PROM, spontaneous abortion and abruptio placentae. Best Pract Res Clin Obstet Gynaecol 2011, 25:313-327

4. Redman CW, Sargent IL: Placental stress and pre-eclampsia: a revised view. Placenta 2009, 30:38-42

5. Roberts JM, Hubel CA: The two stage model of preeclampsia: variations on the theme. Placenta 2009, 30:32-37

6. Steegers EAP, von Dadelszen P, Duvekot JJ, Pijnenborg R: Preeclampsia. Lancet 2010, 376:631-644

7. Roberts JM, Hubel CA: Is oxidative stress the link in the two-stage model of pre-eclampsia? Lancet 1999, 354:788-789

8. Myatt L, Cui X: Oxidative stress in the placenta. Histochem Cell Biol 2004, 122:369-382

9. Heazell AEP, Sharp AN, Baker PN, Crocker IP: Intra-uterine growth restriction is associated with increased apoptosis and altered expression of proteins in the p53 pathway in villous trophoblast. Apoptosis 2011, 16:135-144

10. Leung DN, Smith SC, To KF, Sahota DS, Baker PN: Increased placental apoptosis in pregnancies complicated by preeclampsia. Am J Obstet Gynecol 2001, 184:1249-1250

11. Velauthar L, Plana MN, Kalidindi M, Zamora J, Thilaganathan B, Illanes SE, Khan KS, Aquilina J, Thangaratinam S: Uterine artery Doppler in the first trimester as a risk factor for adverse pregnancy outcomes: a meta-analysis involving 55,974 women. Ultrasound Obstet Gynecol 2014, 43:500-507

12. Kleinrouweler CE, Bossuyt PM, Thilaganathan B, Vollebregt KC, Arenas Ramirez J, Ohkuchi A, Deurloo KL, Macleod M, Diab AE, Wolf H, van der Post JA, Mol BW, Pajkrt E: Value of adding secondtrimester uterine artery Doppler to patient characteristics in identification of nulliparous women at increased risk for pre-eclampsia: an individual patient data meta-analysis. Ultrasound Obstet Gynecol 2013, 42:257-267

13. Melchiorre K, Leslie K, Prefumo F, Bhide A, Thilaganathan B: Firsttrimester uterine artery Doppler indices in the prediction of smallfor-gestational age pregnancy and intrauterine growth restriction. Ultrasound Obstet Gynecol 2009, 33:524-529

14. Melchiorre K, Wormald B, Leslie K, Bhide A, Thilaganathan B: First-trimester uterine artery Doppler indices in term and preterm preeclampsia. Ultrasound Obstet Gynecol 2008, 32:133-137

15. Verlohren S, Melchiorre K, Khalil A, Thilaganathan B: Uterine artery Doppler, birth weight and timing of onset of pre-eclampsia: providing insights into the dual etiology of late-onset pre-eclampsia. Ultrasound Obstet Gynecol 2014, 44:293-298

16. Prefumo F, Sebire NJ, Thilaganathan B: Decreased endovascular trophoblast invasion in first trimester pregnancies with highresistance uterine artery Doppler indices. Hum Reprod 2004, 19: 206-209

17. Whitley GSJ, Dash PR, Ayling L-J, Prefumo F, Thilaganathan B, Cartwright JE: Increased apoptosis in first trimester extravillous trophoblasts from pregnancies at higher risk of developing preeclampsia Am J Pathol 2007, 170:1903-1909

18. Fraser R, Whitley GS, Johnstone AP, Host AJ, Sebire NJ Thilaganathan B, Cartwright JE: Impaired decidual natural killer cell regulation of vascular remodelling in early human pregnancies with high uterine artery resistance. J Pathol 2012, 228:322-332

19. Hollis B, Mavrides E, Campbell S, Tekay A, Thilaganathan B: Reproducibility and repeatability of transabdominal uterine artery Doppler velocimetry between 10 and 14 weeks of gestation. Ultrasound Obstet Gynecol 2001, 18:593-597

20. Brown MA, Lindheimer MD, de Swiet M: The classification and diagnosis of the hypertensive disorders of pregnancy: statement from the International Society for the Study of Hypertension in Pregnancy (ISSHP). Hypertens Pregnancy 2001, 20:IX-XIV

21. Hollis B, Prefumo F, Bhide A, Rao S, Thilaganathan B: Firsttrimester uterine artery blood flow and birth weight. Ultrasound Obstet Gynecol 2003, 22:373-376

22. Napolitano R, Melchiorre K, Arcangeli T, Dias T, Bhide A, Thilaganathan B: Screening for pre-eclampsia by using changes in uterine artery Doppler indices with advancing gestation. Prenat Diagn 2012, 32:180-184

23. Napolitano R, Rajakulasingam R, Memmo A, Bhide A Thilaganathan B: Uterine artery Doppler screening for pre-eclampsia: comparison of the lower, mean and higher first-trimester pulsatility indices. Ultrasound Obstet Gynecol 2011, 37:534-537

24. Prefumo F, Fratelli N, Ganapathy R, Bhide A, Frusca T, Thilaganathan B: First trimester uterine artery Doppler in women with previous pre-eclampsia. Acta Obstet Gynecol Scand 2008, 87: $1271-1275$

25. Grant I, Cartwright JE, Lumicisi B, Wallace AE, Whitley GS: Caffeine inhibits EGF-stimulated trophoblast cell motility through the inhibition of mTORC2 and Akt. Endocrinology 2012, 153:4502-4510

26. Carbon S, Ireland A, Mungall CJ, Shu S, Marshall B, Lewis S AmiGO Hub, Web Presence Working Group. AmiGO: Online access to ontology and annotation data. Bioinformatics 2009, 25:288-289

27. Iacovella C, Franchi M, Egbor M, Bhide A, Thilaganathan B: Relationship of first-trimester uterine artery Doppler to late stillbirth. Prenat Diagn 2012, 32:557-561

28. Enquobahrie DA, Meller M, Rice K, Psaty BM, Siscovick DS, Williams MA: Differential placental gene expression in preeclampsia. Am J Obstet Gynecol 2008, 199:566.e1-566.e11 
29. Sitras V, Paulssen RH, Grønaas H, Leirvik J, Hanssen TA, Vårtun A, Acharya G: Differential placental gene expression in severe preeclampsia. Placenta 2009, 30:424-433

30. Founds SA, Conley YP, Lyons-Weiler JF, Jeyabalan A, Allen Hogge W, Conrad KP: Altered global gene expression in first trimester placentas of women destined to develop preeclampsia. Placenta 2009, 30:15-24

31. Farina A, Morano D, Arcelli D, De Sanctis P, Sekizawa A, Purwosunu Y, Zucchini C, Simonazzi G, Okai T, Rizzo N: Gene expression in chorionic villous samples at 11 weeks of gestation in women who develop preeclampsia later in pregnancy: implications for screening. Prenat Diagn 2009, 29:1038-1044

32. Semenza GL, Nejfelt MK, Chi SM, Antonarakis SE: Hypoxia-inducible nuclear factors bind to an enhancer element located $3^{\prime}$ to the human erythropoietin gene. Proc Natl Acad Sci U S A 1991, 88:5680-5684

33. Caniggia I, Winter J, Lye SJ, Post M: Oxygen and placental development during the first trimester: implications for the pathophysiology of pre-eclampsia. Placenta 2000, 21(Suppl A):S25-S30

34. Soleymanlou N, Jurisica I, Nevo O, Ietta F, Zhang X, Zamudio S, Post M, Caniggia I: Molecular evidence of placental hypoxia in preeclampsia. J Clin Endocrinol Metab 2005, 90:4299-4308

35. Rolfo A, Many A, Racano A, Tal R, Tagliaferro A, Ietta F, Wang J, Post M, Caniggia I: Abnormalities in oxygen sensing define early and late onset preeclampsia as distinct pathologies. PLoS One 2010, 5:0013288

36. Myatt L, Webster RP: Vascular biology of preeclampsia. J Thromb Haemost 2009, 7:375-384

37. Belo L, Caslake M, Santos-Silva A, Castro EMB, Pereira-Leite L, Quintanilha A, Rebelo I: LDL size, total antioxidant status and oxidised LDL in normal human pregnancy: a longitudinal study. Atherosclerosis 2004, 177:391-399

38. Jauniaux E, Watson AL, Hempstock J, Bao YP, Skepper JN, Burton GJ: Onset of maternal arterial blood flow and placental oxidative stress: a possible factor in human early pregnancy failure. Am J Pathol 2000, 157:2111-2122

39. Burton GJ, Jauniaux E: Placental oxidative stress: from miscarriage to preeclampsia. J Soc Gynecol Investig 2004, 11:342-352

40. Vanderlelie J, Venardos K, Clifton VL, Gude NM, Clarke FM, Perkins AV: Increased biological oxidation and reduced anti-oxidant enzyme activity in pre-eclamptic placentae. Placenta 2005, 26:53-58

41. Mistry HD, Wilson V, Ramsay MM, Symonds ME, Broughton Pipkin F: Reduced selenium concentrations and glutathione peroxidase activity in preeclamptic pregnancies. Hypertension 2008, 52: 881-888

42. Levy R, Smith SD, Yusuf K, Huettner PC, Kraus FT, Sadovsky Y, Nelson DM: Trophoblast apoptosis from pregnancies complicated by fetal growth restriction is associated with enhanced p53 expression. Am J Obstet Gynecol 2002, 186:1056-1061

43. Leslie K, Thilaganathan B, Papageorghiou A: Early prediction and prevention of pre-eclampsia. Best Pract Res Clin Obstet Gynaecol 2011, 25:343-354

44. Hubel CA, Roberts JM, Taylor RN, Musci TJ, Rogers GM, McLaughlin MK: Lipid peroxidation in pregnancy: new perspectives on preeclampsia. Am J Obstet Gynecol 1989, 161:1025-1034

45. Huppertz B, Weiss G, Moser G: Trophoblast invasion and oxygenation of the placenta: measurements versus presumptions. J Reprod Immunol 2014, 101-102:74-79
46. Wang GL, Jiang BH, Rue EA, Semenza GL: Hypoxia-inducible factor 1 is a basic-helix-loop-helix-PAS heterodimer regulated by cellular O2 tension. Proc Natl Acad Sci U S A 1995, 92:5510-5514

47. Jauniaux E, Hempstock J, Greenwold N, Burton GJ: Trophoblastic oxidative stress in relation to temporal and regional differences in maternal placental blood flow in normal and abnormal early pregnancies. Am J Pathol 2003, 162:115-125

48. Pringle KG, Kind KL, Thompson JG, Roberts CT: Complex interactions between hypoxia inducible factors, insulin-like growth factor-II and oxygen in early murine trophoblasts. Placenta 2007, 28:1147-1157

49. Araki-Taguchi M, Nomura S, Ino K, Sumigama S, Yamamoto E, Kotani-Ito T, Hayakawa H, Kajiyama H, Shibata K, Itakura A, Kikkawa F: Angiotensin II mimics the hypoxic effect on regulating trophoblast proliferation and differentiation in human placental explant cultures. Life Sci 2008, 82:59-67

50. Pringle KG, Kind KL, Sferruzzi-Perri AN, Thompson JG, Roberts CT: Beyond oxygen: complex regulation and activity of hypoxia inducible factors in pregnancy. Hum Reprod Update 2010, $16: 415-431$

51. Wallace AE, Host AJ, Whitley GS, Cartwright JE: Decidual natural killer cell interactions with trophoblasts are impaired in pregnancies at increased risk of preeclampsia. Am J Pathol 2013, 183:1853-1861

52. Rajakumar A, Conrad KP: Expression, ontogeny, and regulation of hypoxia-inducible transcription factors in the human placenta. Biol Reprod 2000, 63:559-569

53. Koh MY, Powis G: Passing the baton: the HIF switch. Trends Biochem Sci 2012, 37:364-372

54. Patel J, Landers K, Mortimer RH, Richard K: Regulation of hypoxia inducible factors (HIF) in hypoxia and normoxia during placental development. Placenta 2010, 31:951-957

55. Strålin P, Marklund SL: Multiple cytokines regulate the expression of extracellular superoxide dismutase in human vascular smooth muscle cells. Atherosclerosis 2000, 151:433-441

56. Sharp AN, Heazell AEP, Crocker IP, Mor G: Placental apoptosis in health and disease. Am J Reprod Immunol 2010, 64:159-169

57. Crocker IP, Cooper S, Ong SC, Baker PN: Differences in apoptotic susceptibility of cytotrophoblasts and syncytiotrophoblasts in normal pregnancy to those complicated with preeclampsia and intrauterine growth restriction. Am J Pathol 2003, 162:637-643

58. Kingdom J, Huppertz B, Seaward G, Kaufmann P: Development of the placental villous tree and its consequences for fetal growth. Eur J Obstet Gynecol Reprod Biol 2000, 92:35-43

59. Forbes K, Westwood M, Baker PN, Aplin JD: Insulin-like growth factor I and II regulate the life cycle of trophoblast in the developing human placenta. Am J Physiol Cell Physiol 2008, 294:C1313-C1322

60. Mayhew TM: Turnover of human villous trophoblast in normal pregnancy: what do we know and what do we need to know? Placenta 2014, 35:229-240

61. Harris LK, Crocker IP, Baker PN, Aplin JD, Westwood M: IGF2 actions on trophoblast in human placenta are regulated by the insulinlike growth factor 2 receptor, which can function as both a signaling and clearance receptor. Biol Reprod 2011, 84:440-446

62. Longtine MS, Chen B, Odibo AO, Zhong Y, Nelson DM: Villous trophoblast apoptosis is elevated and restricted to cytotrophoblasts in pregnancies complicated by preeclampsia, IUGR, or preeclampsia with IUGR. Placenta 2012, 33:352-359 